\title{
EDITORIAL
}

Animal models

\section{Use of high-fat diets to study rodent obesity as a model of human obesity}

\author{
John R. Speakman (10)
}

Received: 16 January 2019 / Accepted: 20 January 2019 / Published online: 9 April 2019

(c) Springer Nature Limited 2019

The main focus of the International Journal of Obesity (IJO) is to better understand human obesity. Many scientists use rodents as convenient models to dissect aspects of physiology and body-weight control that would not be feasible in humans, such as invasive and terminal investigations. At present there is a range of commercial high-fat diets available that have been demonstrated to make small rodents obese. However, some of these diets contain levels of dietary fat that are much higher than the levels that humans routinely consume. The question has been raised as to whether experimental use of these diets with very high levels of fat adequately models the situation of human obesity. If not, the question arises whether the IJO should continue to accept such papers for publication. The Editorial Board of IJO was asked its views on this issue: 14 Board members replied (listed at the end). This short editorial summarises those responses.

Several of the responses came from clinical scientists with no direct experience of performing work on rodents. Together they all reinforced the view that if rodent models can be made more closely to mimic what happens in humans then it is likely that the insights following from such work will be enhanced. There was also a strong feeling, however, that while the journal might advise what they think is best, they should not impose a ban on future papers for these methodological reasons.

Many of the basic scientists with experience of using rodent models emphasised the reason and history behind the choice of diets containing $60 \%$ fat to create obesity in mice. At the moment the biggest supplier of rodent diets is the

John R. Speakman

j.speakman@abdn.ac.uk

1 State Key Laboratory of Molecular Developmental Biology, Institute of Genetics and Developmental Biology, Chinese

Academy of Sciences, Beijing, Center for Developmental Biology No. 1 West Beichen Road, Chaoyang District, Beijing 100101, China company Research Diets. About 20 years ago they created a series of 3 diets known as the DIO series. These had, respectively $10 \%, 45 \%$ and $60 \%$ fat by calories. A normal rodent diet contains about $10 \%$ fat, so both 45 and $60 \%$ fat diets are high-fat for rodents. Mice on the $45 \%$ fat diet become obese. However, mice on the $60 \%$ fat diet become more obese, and do so more rapidly. This is advantageous because it reduces the time that the animals need to be housed, thereby reducing caging costs. Many researchers therefore use the $60 \%$ rodent diet as a matter of economics and convenience. Some commercial suppliers of prefattened mice also use this diet to accelerate the fattening process and reduce the costs. The typical American or European diet, however, contains about $36-40 \%$ fat by energy, so a tolerable high-fat human diet might contain $50-60 \%$ of energy as fat. However, the $60 \%$ fat rodent diet presents a much greater distortion of the fat content of a normal rodent chow. Thus rodent studies with a $60 \%$ fat content are potentially not as relevant to human physiology as those which use a $45 \%$ fat diet.

A key question then is whether there is any difference in the resultant obesity, physiology and metabolism of the mice fed high (c. 45\%) vs. very high (c. 60\%) fat diets. Several of the responses from the board addressed this issue directly and the answer seems to be that very high fat diets produce a more exaggerated metabolic response than highfat diets, but the difference is relatively small. For example, the extent of obesity and impacts on glucose homoeostasis and insulin resistance increase with increasing fat content up to around $60 \%$ fat $[1,2]$. Although some studies have failed to detect such differences [3, 4]. Impacts on cognition with age appear relatively minor $[4,5]$. A comparison of metabolite profiles of low-fat (10\%) vs. high-fat diet $(45 \%$ fat) vs. very high-fat diet ( $60 \%$ fat) fed mice revealed that 80 metabolites were altered in the lungs of high-fat fed mice relative to the control diet [6]. However, only 35 of these 80 changed metabolites were common across the two high-fat diets, indicating these diets generated rather different metabolic responses. This is not an exhaustive review but it 
does show in many cases the impact of high vs. very high diets is often small, but it can occasionally be much larger. A caveat however is that given there are also differences in sucrose content between these diets, and the type of fat is also not constant (see below) the contrasts may have been different had these confounding factors been controlled.

A couple of the responses concerned the issue that the DIO series of diets differ not only in their fat content but also in other components that may be important in terms of driving effects on metabolic health. The $60 \%$ diet for example has substantially less sucrose than the $45 \%$ diet, which in turn has less than the original $10 \%$ fat diet. This inverse relation of fat to sucrose might be an issue given the much elevated recent interest in the links of sugar consumption to metabolic health (but see ref. [2]). However, research diets have responded to these concerns over sugar contents by producing additional versions of the control diet that match the sucrose levels of the 45 and $60 \%$ diets. This emphasises the importance of detailing very precisely in the methods sections of papers exactly what control diets were used, as the comparisons to controls may be very different if the control group has had 35\% sucrose (the original 10\% diet) compared to $7 \%$ (10\% fat diet matched to the $60 \%$ fat for sucrose level).

Finally, if we are concerned about making the diets we feed to rodents match more closely the diets eaten by humans then we should probably also consider not only the $\%$ fat in the diet but also its fatty acid (FA) composition. It is well known that dietary FAs can influence the level of obesity [7]. Yet, none of the DIO series from research diets mimics closely the FA composition of the human diet, and they are also inconsistent with each other across the different levels of fat. Diets from other companies also use a variety of FA compositions that are not designed to mimic the human diet, and also differ from the DIO series.

To overcome this latter issue Research Diets recently created a series of diets that vary in their macronutrient contents, but all of them have the same dietary fat composition [2]. This composition has been matched to the saturated/mono-unsaturated/polyunsaturated composition of the standard American diet, and has an n-3:n-6 ratio of 1:14.7 that also mimics the situation in the human diet. There are 30 diets in total and they cover a broad range of macronutrient contents from 5 to $30 \%$ protein, 8.3 to $80 \%$ fat and 10 to $80 \%$ carbohydrate, including variation from 5 to $30 \%$ sucrose. Some of these diets closely mimic the level and composition of fat in the human diet, and can be compared to control diets with a lower fat $\%$ but the exact same fatty acid composition, with sucrose levels high or low. These diets are available on the Research Diets website https://www.researchdiets.com/blog/posts/macronutrientdiets and provide a resource for researchers wishing to mimic more closely the dietary habits associated with obesity in humans.

Overall, the message from the board of IJO was that we encourage researchers to try and mimic in rodent studies aspects of the diet that are similar to those found in humans, in the hope this will provide better insights into the causes and consequences of obesity.

Acknowledgements The board members who responded to the call for information that informed the above editorial were Hans-Rudi Berthoud, Weiqin Chen, Osamu Ezaki, Nick Finer, Frank Greenaway, Joan Han, David Heal, Andrew Hill, John Kirwan, Mike Lean, David Stensel, John Speakman, Angelo Tremblay and Licio Velluso.

\section{Compliance with ethical standards}

Conflict of interest The authors declare that they have no conflict of interest.

Publisher's note: Springer Nature remains neutral with regard to jurisdictional claims in published maps and institutional affiliations.

\section{References}

1. Takahashi M, Ikemoto S, Ezaki O. Effect of the fat/carbohydrate ratio in the diet on obesity and oral glucose tolerance in C57BL/6J mice. J Nutr Sci Vitaminol. 1999;45:583-93.

2. Hu SM, Wang L, Yang DB, Li L, Togo J, Wu YG, et al. Dietary fat, but not dietary protein or carbohydrate, regulates energy intake and causes adiposity in mice. Cell Metab. 2018;28:415-31.

3. Mundy AL, Haas E, Bhattacharya I, Widmer CC, Kretz M, Hofmann-Lehmann R, Minotti R, Barton M. Fat intake modifies vascular responsiveness and receptor expression of vasoconstrictors: implications for diet induced obesity. Cardiovasc Res. 2007;73:368-75.

4. Morrison CD, Pistell PJ, Ingram DK, Johnson WD, Liu Y, et al. High fat diet increases hippocampal oxidative stress and cognitive impairment in aged mice: implications for decreased Nrf2 signalling. J Neurochem. 2010;114:1581-9.

5. Arnold SE, Lucki I, Brookshire B,R, et al. High fat diet produces brain insulin resistance, synaptodendritic abnormalities and altered behaviour in mice. Neurobiol Dis. 2014;67:79-87.

6. Showalter MR, Nonnecke EB, Linderholm AL, et al. Obesogenic diets alter metabolism in mice. PLoS ONE. 2018;13:e0190632.

7. Nakatani T, Kim HJ, Kaburagi Y, Yasuda K, Ezaki O. A low fish oil inhibits SREBP-1 proteolytic cascade, while a high-fish-oil feeding decreases SREBP-1 mRNA in mice liver: relationship to anti-obesity. J Lipid Res. 2003;44:369-79. 